\title{
Research Paper \\ The Effect of Omega-3 Supplementation and Functional Exercises on the Psychomotor Performance of Aged Women in Qazvin
}

\author{
*Morteza Taheri ${ }^{1}$ (C), Khadijeh Irandoust ${ }^{1}$ (i)
}

1. Department of Physical Education and Sports Sciences, Faculty of Social Sciences, Imam Khomeini International University, Qazvin, Iran.

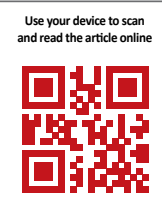

Chat on Taheri M, Irandoust Kh. [The Effect of Omega-3 Supplementation and Functional Exercises on the Psychomotor Performance of Aged Women in Qazvin (Persian)]. Salmand: Iranian Journal of Ageing. 2019; 14(1):2-13. https://doi.org/10.32598/sija.13.10.100

https://doi.org/10.32598/sija.13.10.100

Received: 10 Oct 2018 Accepted: 23 Jan 2019 Available Online: 01 Apr 2019

Key words: Exercise, Aging, Quality of life

\begin{abstract}
A B STRACT
Objectives Older age and its associated biopsychological disorders exert socioeconomic costs. Therefore, the importance of preventing and treating these situations is important for researchers and authorities. Therefore, the present study investigated the effects of omega-3 supplementation and functional exercises on the psychomotor performance of aged women.

Methods \& Materials This was a semi-experimental study with a Pre-test-Post-test design. In total, 47 aged women were recruited based on convenience sampling method from the Sports and Health Consultation Center of Qazvin Sports and Youth Department. The subjects were randomly divided into functional exercises, omega-3 supplement, exercise+supplement, and placebo groups. The functional exercise protocol consisted of 8 weeks; 3 sessions per week (each session lasted $50 \mathrm{~min}$ ). Food analysis was performed to control the possible effect of nutrition on the obtained results. Psychomotor performance was measured using the Vienna universal system. The obtained data were analyzed using multivariate analysis of covariance.

Results The attained results suggested that exercise and omega- 3 could improve all psychomotor performance test scores $(P \leq 0.05)$. Furthermore, functional exercises and supplement group demonstrated a significant improvement in the tests after the intervention, compared to the control group $(P \leq 0.05)$.

Conclusion The exercise and omega-3 program could improve psychomotor performance, leading to improved quality of life. Consuming omega-3 and functional exercises are highly recommended for improving the psychomotor performance of aged women.
\end{abstract}

\section{Extended Abstract}

\section{Objectives}

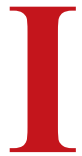

n a 100-year interval, the number of elderly people aged $\geq 60$ years has been tripled. Furthermore, it is estimated that the aged population will increase from $11 \%$ of the total world population in 1950 to $30 \%$ of that by 2050 [1]. Changes occurring in various body systems cause mobility limitations in aged people and impair their functional independence [2]. Evidence suggests abnormalities in the psychomotor performance of the elderly, which may lead to more impaired functional independence. One of these effective indicators is the information processing ability that includes factors such as reaction

\section{* Corresponding Author:}

Morteza Taheri, PhD.

Address: Department of Physical Education and Sports Sciences, Faculty of Social Sciences, Imam Khomeini International University, Qazvin, Iran Tel: +98 (912) 4070721

E-mail: m.taheri@soc.ikiu.ac.ir 
time, attention capacity, and ability to respond to environmental stimuli [4]. The present study aimed to investigate the effect of omega-3 supplementation and functional exercises on the psychomotor performance of aged women.

\section{Methods and Materials}

This was a quasi-experimental study with Pre-testPost-test design conducted in 2017 on 47 elderly women aged $60-70$ years. The study participants were recruited from the Sports and Health Consultation Center of Qazvin Sports and Youth Department based on convenience sampling method. They were randomly assigned to 4 groups of functional exercises (EX), omega-3 supplementation (SUP), exercise+omega-3 supplementation (EX+SUP), and placebo. Inclusion criteria were the age of $\geq 60$ years, not susceptible to omega-3 or fish supplements' adverse effects, the ability to participate in training exercises, and no mobility limitations. To assess the readiness of subjects during training, the Physical Activity Readiness Questionnaire (PAR-Q) was used.

The nutritional analysis of study subjects was examined before testing by N4 software to control the effect of nutrition on the results. The two groups of SUP and EX+SUP received two $2000 \mathrm{mg}$ of omega- 3 capsules (EPA 180 and DHA 120, Zahrawi Co.) daily for 8 weeks in the morning and night. The placebo group received two capsules containing $2 \%$ dextrose solution (Zakaria Co.). The functional exercise protocol was designed according to the American College of Sports Medicine instructions, consisting of 3 sessions/week (Saturday, Monday and Wednesday) for $50 \mathrm{~min}$ from 10:00 AM to 11:30 AM, and at least 1 hour after breakfast. Each session included a 10-min warm up, a 45-min strength and endurance training, and a 5-min cool down [5].

Psychomotor performance was measured using the Vienna test system which has acceptable validity. One- way Analysis of Variance (ANOVA) and Multivariate Analysis of Covariance (MANCOVA) were used for analyzing the collected data. Descriptive statistics of study groups before and after the intervention are presented in Table 1.

\section{Results}

The Smirnov-Kolmogorov test results suggested the normal distribution of the obtained data $(\mathrm{P}>0.05)$. Oneway ANOVA used for comparing the nutritional status of subjects reported no significant difference on the day before the pre-test phase $(\mathrm{P}>0.05)$. Groups EX, SUP, and EX-SUP demonstrated significant improvements in all psychomotor tests $(\mathrm{P}<0.05)$. By performing repeated measures ANOVA on three components in the 4 groups, a significant difference between the mean Pre-test and Post-test scores was observed $(\mathrm{P}<0.05)$. By removing the Pre-test effect, it was revealed that when a combination of omega-3 supplementation and functional exercises was used, the best results were achieved and their psychomotor performance was improved.

\section{Conclusion}

Any exercise intervention addressing aged people with mental challenges in terms of information processing and leading them to the problem-solving process can enhance their psychomotor performance. Based on the obtained results, the study participants' response and precision of functional performance improved after exercise and supplementation interventions; this finding is very important in the motor control of aged people. This study reported improved psychomotor performance following functional exercising and omega-3 supplementation which should be considered by policymakers.

Table 1. Descriptive statistics of study groups before and after the intervention

\begin{tabular}{|c|c|c|c|c|c|c|c|c|c|}
\hline \multirow{3}{*}{ Variable } & \multirow{3}{*}{ Test } & \multicolumn{8}{|c|}{ Mean \pm SD } \\
\hline & & \multicolumn{2}{|c|}{ EX $(n=13)$} & \multicolumn{2}{|c|}{ SUP (n=12) } & \multicolumn{2}{|c|}{ EX+SUP (n=12) } & \multicolumn{2}{|c|}{ Placebo (n=12) } \\
\hline & & Pre-test & Post-test & Pre-test & Post-test & Pre-test & Post-test & Pre-test & Post-test \\
\hline \multirow{3}{*}{$\begin{array}{l}\text { Psychomotor } \\
\text { performance }\end{array}$} & Sum hits & $21.1 \pm 2.3$ & $26.1 \pm 2.4$ & $21.1 \pm 4.2$ & $25.1 \pm 7.1$ & $21.1 \pm 7.3$ & $28.1 \pm 2.1$ & $21.1 \pm 2.4$ & $21.1 \pm 6.1$ \\
\hline & $\begin{array}{l}\text { Sum correct } \\
\text { rejection }\end{array}$ & $30.1 \pm 2.1$ & $35 \pm 4.9$ & $31.1 \pm 3.1$ & $34 \pm 4.9$ & $30.1 \pm 8.4$ & $39.1 \pm 2.1$ & $30.1 \pm 4.3$ & $31.1 \pm 2.3$ \\
\hline & $\begin{array}{l}\text { Mean time } \\
\text { hits }\end{array}$ & $1 \pm 88.05$ & $1 \pm 64.04$ & $1 \pm 86.06$ & $1 \pm 68.09$ & $1 \pm 87.03$ & $1 \pm 63.04$ & $1 \pm 84.06$ & $1 \pm 85.04$ \\
\hline
\end{tabular}




\section{Ethical Considerations}

Compliance with ethical guidelines

This study was approved by the Ethics Committee of the International University of Imam Khomeini with the code of 17628 .

\section{Funding}

This research has been extracted from the research project (No. 11821) funded by Imam Khomeini International University.

\section{Authors contributions}

Conceptualization and Methodology: Morteza Taheri; Editing and Finalization: Khadijeh Irandoust.

\section{Conflict of interest}

The authors declared no conflict of interest. 


\title{
تأثير مكمل يارى امكاّـاّ و تمرينات عملكردى بر ظرفيتهاى روانىحركتى زنان سالمند شهر قزوين
}

(1) 'مرتضى طاهرى' (1)، خديجه ايراندوست (

1- كروه علوم ورزشى، دانشكده علوم اجتماعى، دانشكاه بينالملى امام خمينى، قزوين، ايران.

\begin{abstract}
حكند

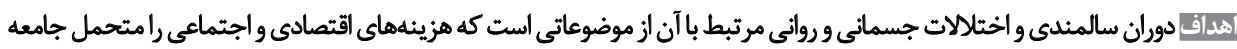

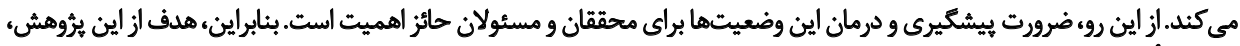

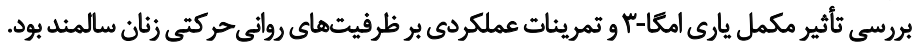

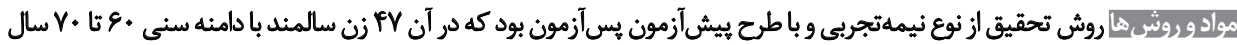

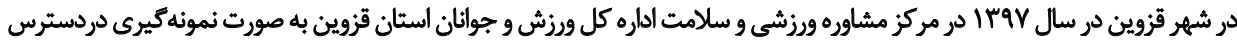

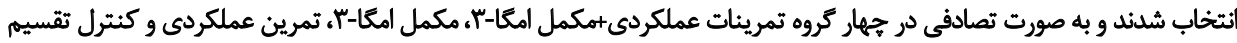

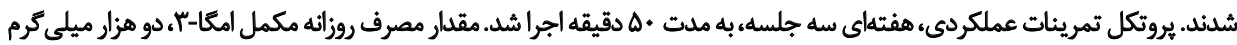

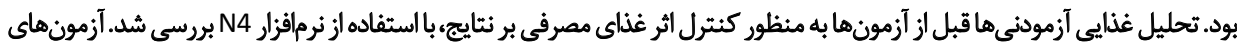

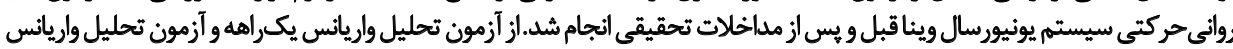

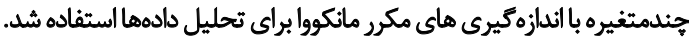

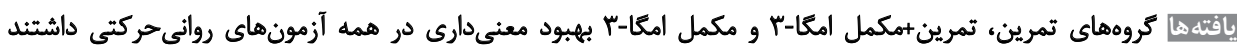

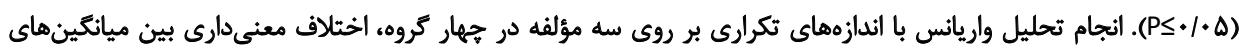

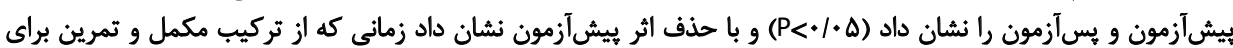

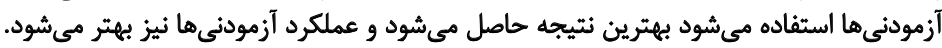

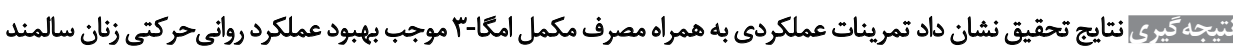

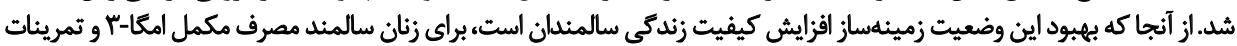

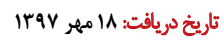

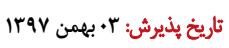

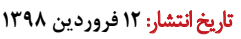

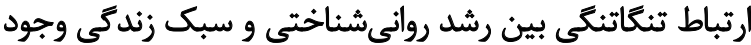

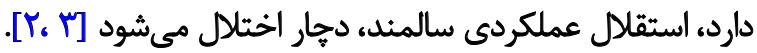

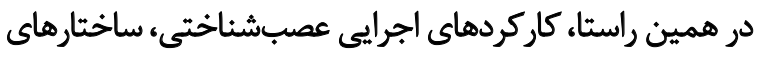

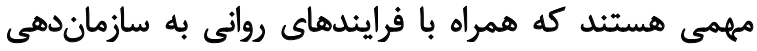

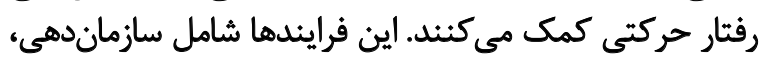

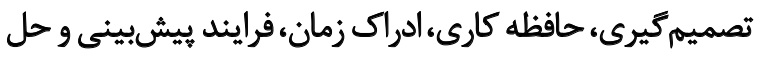

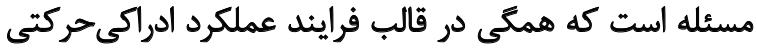

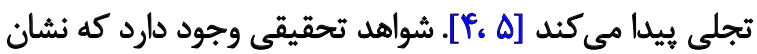

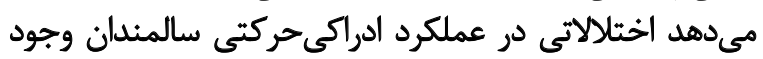

در يك بازه زمانى +. اساله، نسبت افراد سالمند بيش از •ع سال

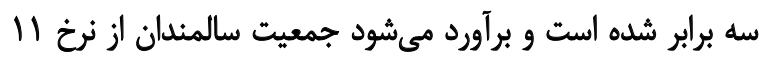

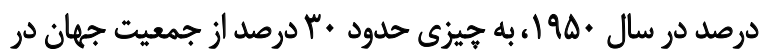

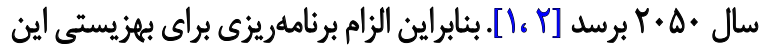

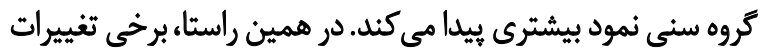

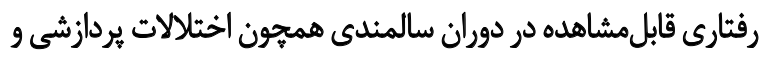

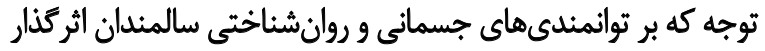

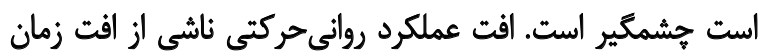

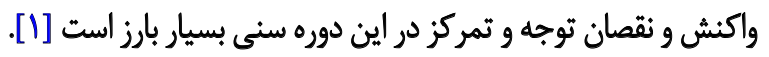


با افت عملكرد شناختى و همينطور عملكرد اجرايى در دوران

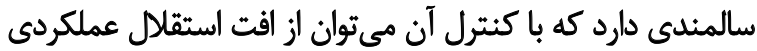

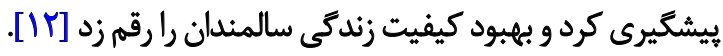
تغييرات فيزيولوزيكى و عملكردى كه در دوران سالمندى

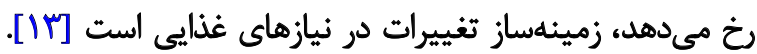

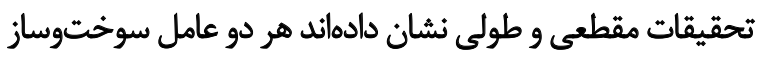

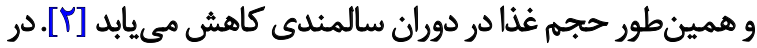

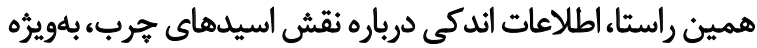

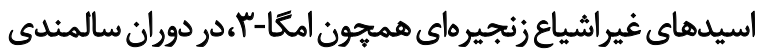

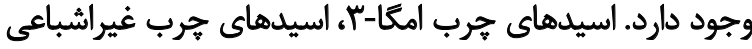

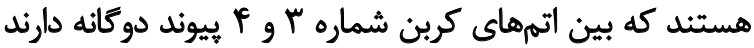

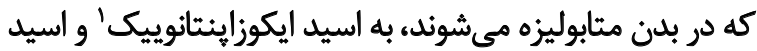

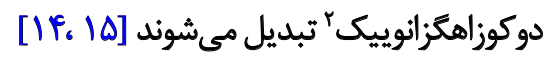

از خواص تثبيتشده اين ماده غذايى ميتوان به ويرتى خدي

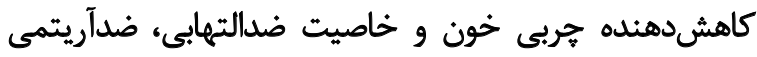

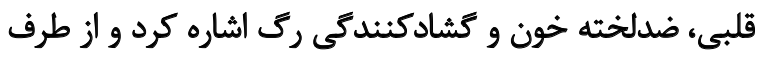

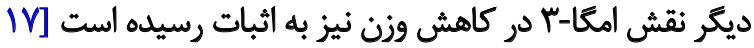

\section{Eicosapentaenoic Acid (EPA)}

2. Docosahexaenoic Acid (DHA)
دارد كه بيشازييش استقلال عملكردى سالمندان را دهار

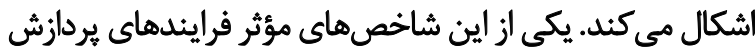

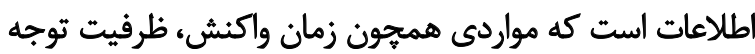

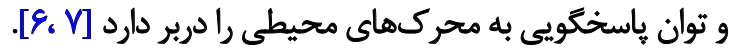
همان طور كه درباره اهميت سبك زندكى سالمندان اشاره

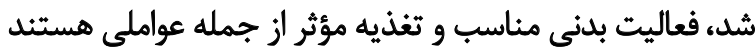

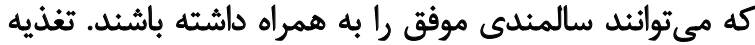

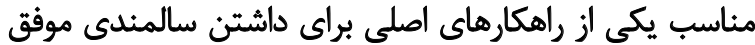

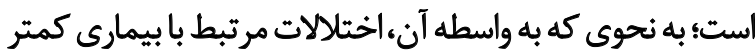

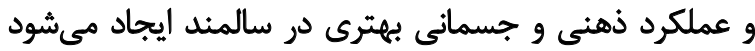

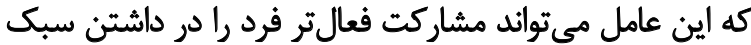

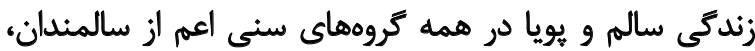

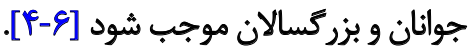
بر اساس مطالعات انجامشده، فقر حركتى و تغذيه نامناسب از

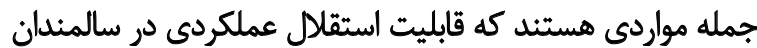

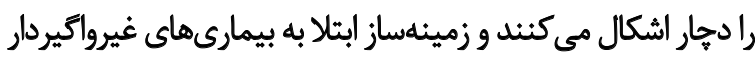

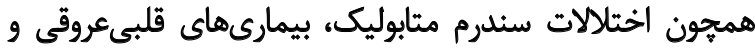

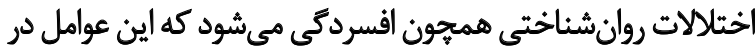

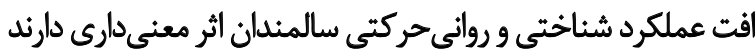

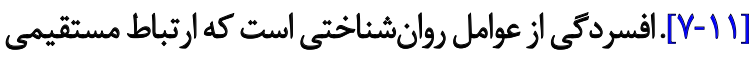

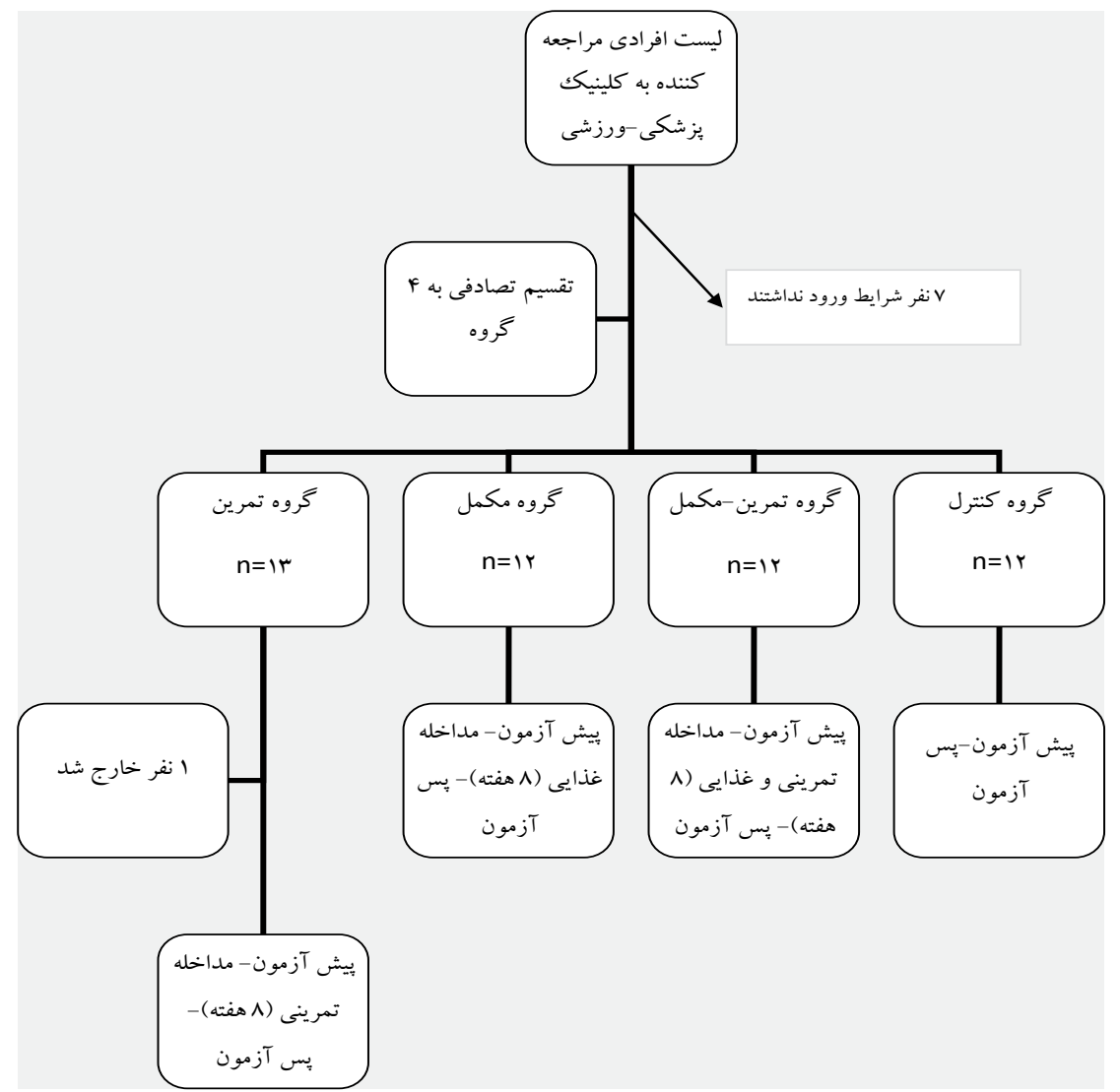


جدول ا. يروتكل تمرينى عملكردى به طور خلاصه

\begin{tabular}{|c|c|}
\hline 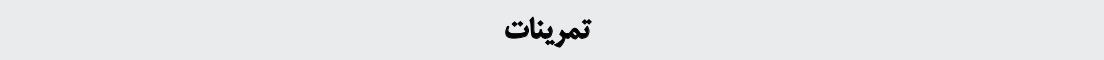 & مرحله (زمان اجرا) \\
\hline راهرفتن و حركات نرمشى و كششى & كرمكردن (•( دقيقه) \\
\hline تمرينات يشت به ديوار: تعادل با يك يا، شناى سوثلى اصلاحشده، تمرينات كرائج، سويرمن، نشسته V، تمرينات ستاره، & 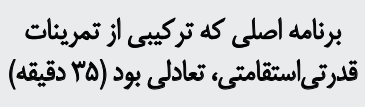 \\
\hline راهرفتن، تمرينات تنفسى و انعطافِذيذي & لسردكردن (ه دقيقه) \\
\hline
\end{tabular}

닌

يرسش نامه آمادكى فعاليت جسمانى إنى

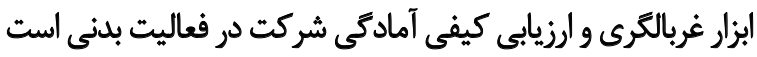

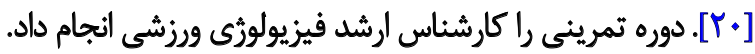

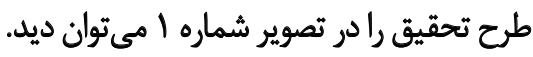

تحليل غذايى آزمودنىها قبل از آزمونها به منظور كنترل اثر

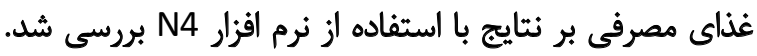

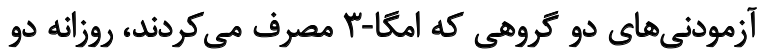
هزار ميلى

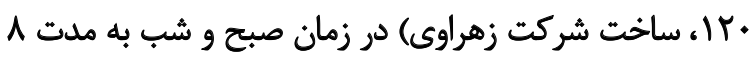

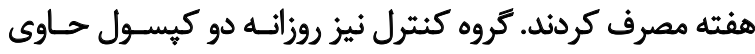

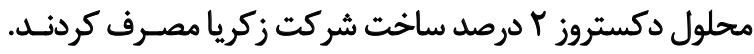

$$
\text { بروتمل تمريني }
$$

يروتكل تمرينى بركرفته از كالج طب ورزش آمريكا" بود كه بـ

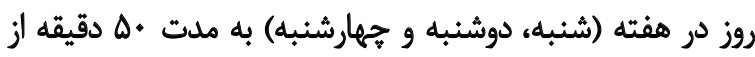

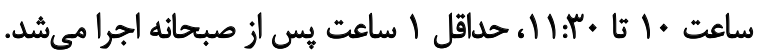

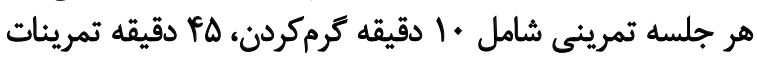

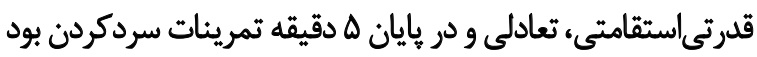

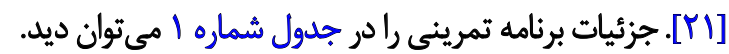

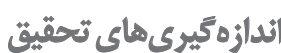

از آنجا كه مصرف ريزمغذىها و درشتمغذىها در روزهاى

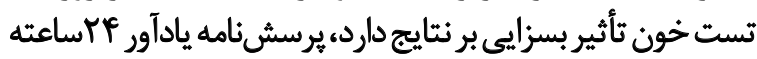

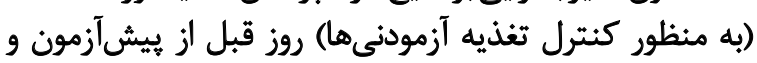
يسآزمون به منظور كنترل اين عامل استفاده شد.

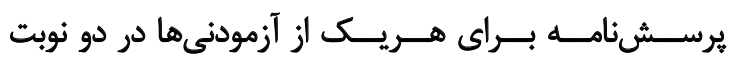

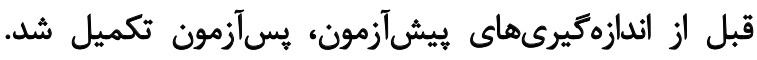

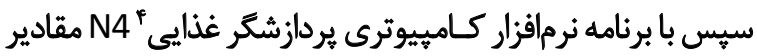

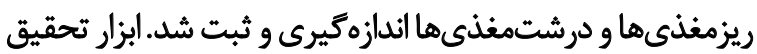
مجموعهابزار وينا بود كه يك صفحه نمايشعر و يكى صفحئه كليد

3. American College of Sport Medicine (ACSM)

4. Food Processor

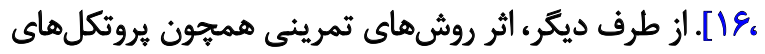

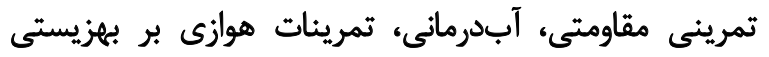

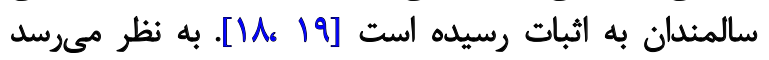

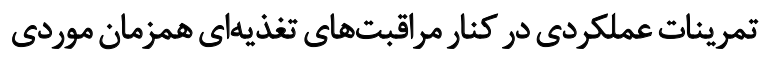

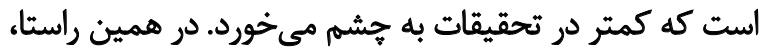

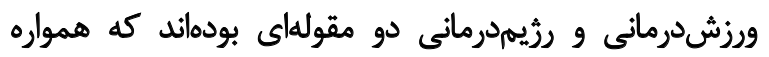

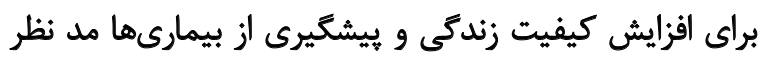

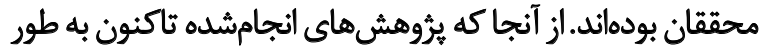

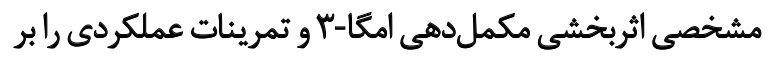

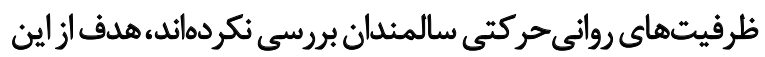

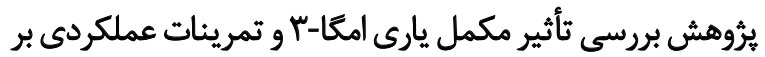

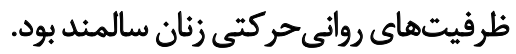
ووش مطاله d

روش تحقيق از نوع نيمهتجربى با طرح بيشآزمون يسآزمون

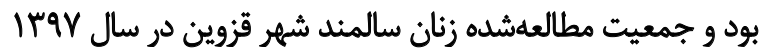

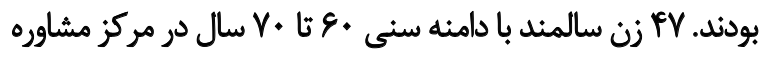

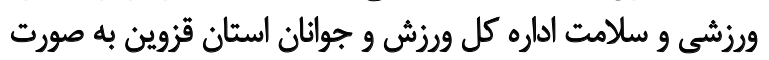

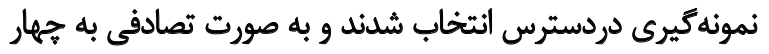

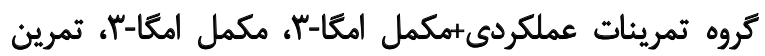

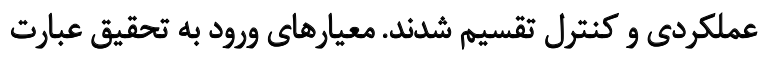

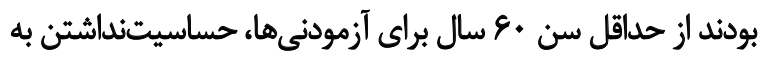

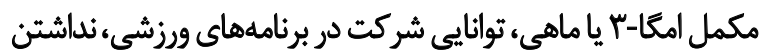

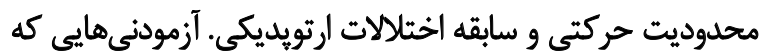

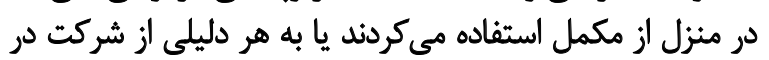

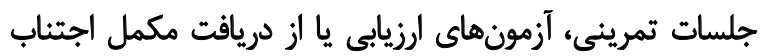
مي كردند از تحقيق كنار كذاشته شدند.

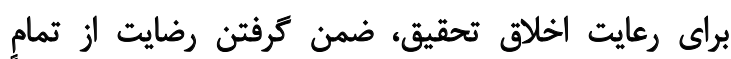

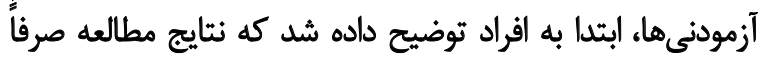

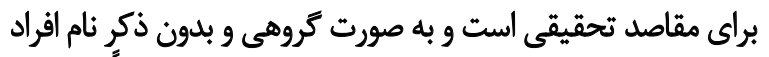

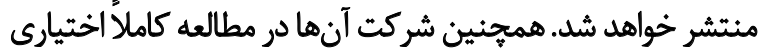

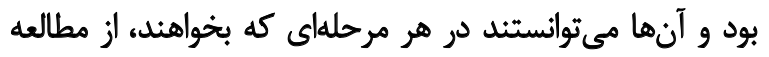

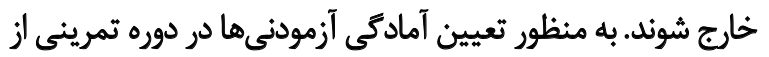


براي مقايسه گروهها در بيش آزمون و يسآزمون از آزمون تحليل

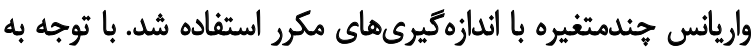

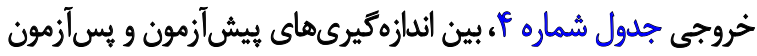

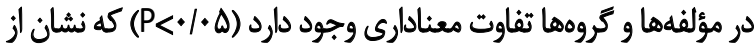

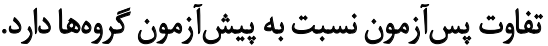

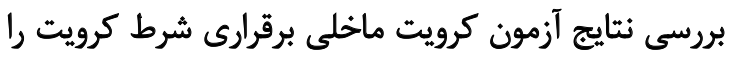

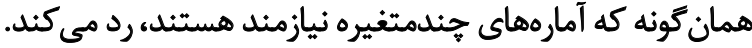

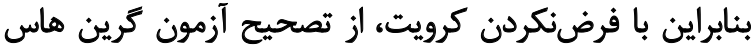

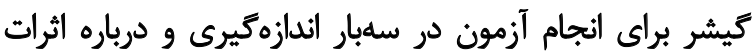

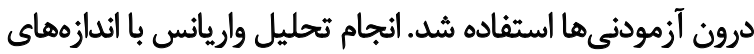

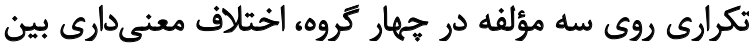

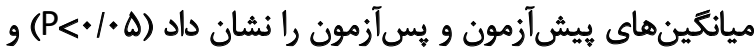

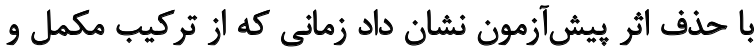

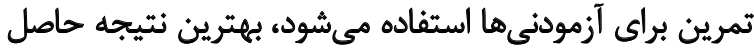
مىشود و عملكرد آزمودنى ها بهتر مى آشود (جدول شماره \&،هاه).

بحث

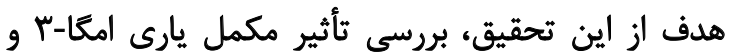

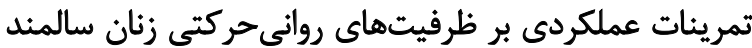

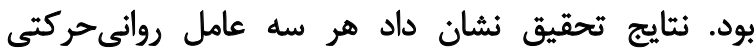

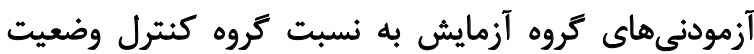

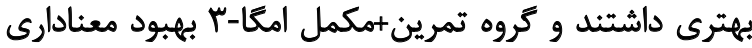

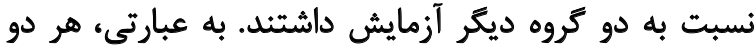

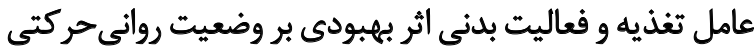
زنان سالمند داشتند. درباره اثربخشى عامل امكا-با، نتايج باليا
شامل دو دسته فلزي است كه به منظور ارزيابى عملكرد ادراكى حركتى

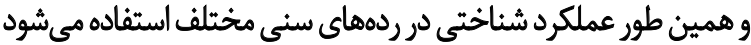

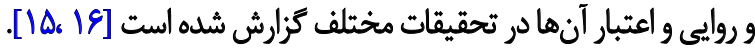

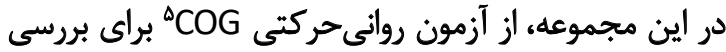

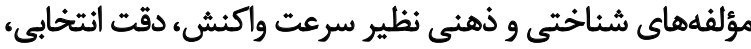

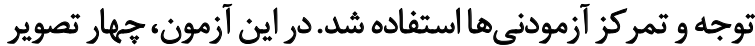

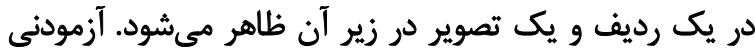

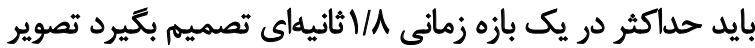

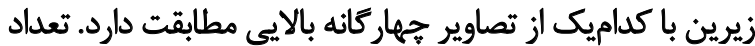

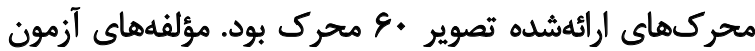

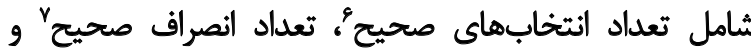

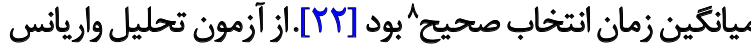

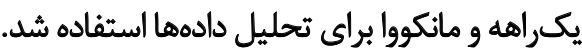

ياثتهها

نتايج آزمون كلموكروف|سميرونوف نشان داد دادهها توزيع

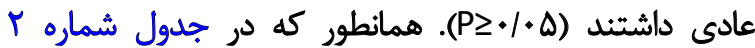

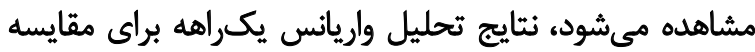

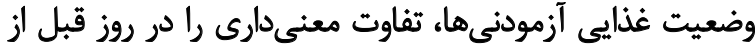

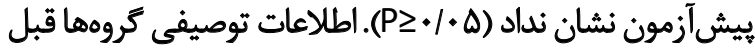

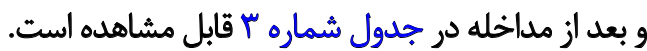

5. Cognitrone

6. Sum hits

7. Sum correct rejection

8. Mean time hits

جدول Y. مقايسه ريزمغذىها و كالرى دريافتى روزانه بين كروهها روز قبل از يسآزمون

\begin{tabular}{|c|c|c|c|c|c|}
\hline $\mathbf{P}$ & كيروه كتترل & تمرينات هوازى +مكمل امكًا-ب & مكمل امكا-r & تمرينات هوازى & كروه \\
\hline.$/ 17 \Delta$ & $|A| \cdot / V \pm f / g$ & $M \cdot \Delta / T^{\top} \pm N \Delta$ & IVQWEET/q & $|A| \backslash / \Delta \pm N E$ & كالرى دريافتى \\
\hline.$/ 11$ & $r \Delta N \mid \pm n$ & $r \Delta I / f \pm E r$ & $r \Delta q / T \pm n$ & $r F \Delta / r \pm F q$ & كربوهيدرات (كرم) \\
\hline.$/ p r$ & $P \backslash / \pm \pm$ & $p+/ f \pm 1 / q$ & $r / / P \pm r / r$ & $r q / \Delta \pm r / r$ & يروتئين (كرم) \\
\hline.$/ 11$ & relate & $r \cdot N \pm P / Q$ & $r \Delta / \Delta \pm \Delta / r^{\circ}$ & $\mathrm{m} / \Lambda \pm E$ & جريى (كرم) \\
\hline .119 & $|E /| \pm \varepsilon$ & $10 / 4 \pm \pi / 4$ & $\mid r / r \pm q /$ & $\mid r / r \pm r / q$ & فيبر (كرم) \\
\hline.$/ 49$ & $e q / 9 \pm \mu$ & $E q \Delta \Delta V / \Lambda$ & $r / F \pm r / \Delta$ & $E N F \pm Y / A$ & كلسترول (ميلى كرم) \\
\hline 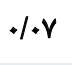 & RENFV $\pm / / A$ & $T M T M E \pm N I$ & YENEVEI/A & $r V \Delta / \Delta \Delta \pm E / \mathcal{A}$ & كلسييم (ميلى كرم) \\
\hline .1 .9 & $\Delta V / \Delta \cdot \pm V / \Lambda$ & $\Delta V / \Delta) \pm \Delta / r^{\mu}$ & $\Delta \Delta / F q \pm V / T$ & $\Delta H / \Delta) \pm V / \Delta$ & ويتامين C (ميلى كرم) \\
\hline.$/ N$ & $r / \Lambda \pm r / r$ & $\Gamma / N+/ N$ & $H / N \pm 1 / \Delta$ & $\vee / \backslash \pm พ / /$ & ويتامين E (ميلىكرم) \\
\hline .101 & $\Delta T / 1 \cdot \pm 1 / 8$ & $\Delta \cdot 1 A \pm r / \Delta$ & $\Delta . / 1 \Delta \pm \Psi / 1$ & $e q / 1 \pm q / 1$ & سلثيوم (ميكروكرم) \\
\hline
\end{tabular}

ill 
جدول r. اطلاعات توصيفى گروهها قبل و بعد از مداخله

\begin{tabular}{|c|c|c|c|c|c|c|c|c|c|}
\hline \multicolumn{2}{|c|}{$\underset{(n=1 Y)}{S}$} & \multicolumn{2}{|c|}{ 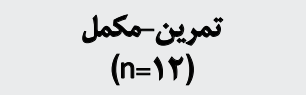 } & \multicolumn{2}{|c|}{ مكمل } & \multicolumn{2}{|c|}{ تمرين } & \multirow{2}{*}{\multicolumn{2}{|c|}{ كروه }} \\
\hline بس أزمون & ييش آزمون & يس أزهون & بيش آزمون & يسآزمون & ييش آزمون & يس أزهون & بيش آزآمون & & \\
\hline$r y / \Lambda \pm g / I$ & $r / / \pm r / H$ & $r N \backslash \pm Y / I$ & $r M / \backslash \pm V / r$ & $r \Delta / / \pm v / /$ & $r / N \pm Y / T$ & $r g / \Lambda \pm r / F$ & $r V / I \pm r / r$ & تعلداد انتخابهاى & $\frac{9}{2}$ \\
\hline$r V / \pm Y / r$ & $r+/ \pm \pm / r$ & $r q / \perp \pm r / /$ & $r \cdot / \pm N e$ & $m / . \pm r / q$ & $r \mathrm{M} / \mathrm{m} / \mathrm{h}$ & $r \bowtie / \bullet \pm \oplus / q$ & $r+/ \pm+r /$ & تعداد انصراف & 7 \\
\hline $1 / 1 \Delta \pm . / \cdot \varphi$ & $V / A F \pm .1 .8$ & $1 / g r \pm .1+4$ & $V / A V \pm=/ * r$ & $1 / 8 \wedge \pm . / \cdot 9$ & $1 / 1 \in \pm \pm .1 \cdot 8$ & Wat $\pm .1+4$ & $V / M \pm+1+\infty$ & صدتزمان انتخاب & \\
\hline
\end{tabular}

乩

جدول F. نتايح آزمون تحليل واريانس جندمتغيره با ائدازهيرىهاى مكرر براى اثرات بين كروهى و مؤلفهها

\begin{tabular}{|c|c|c|c|c|c|c|}
\hline سطح معنادارى & درجه آزادى خطا & درجه آزادى فرضشده & F مقدار F م م & ارزثش & 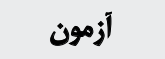 & ثأثير بينكّوهى \\
\hline .10 .1 & $\mu$ & r & $\Delta / \cdot N$ & .1 .94 & لانداى ويلكس & عامل مؤلفهذها \\
\hline $.1+.1$ & $\mu p$ & r & $\theta(A)$ & $\cdot(\cdot r)$ & لامبا ويلكس & تعامل مؤلفهها با كروهها \\
\hline
\end{tabular}

جدول ه. نتايج آزمون ماخلى

\begin{tabular}{|c|c|c|c|}
\hline سطح معنادارى & دوجه أزادى & خي دو & تأثير درون كروهى \\
\hline $.1 \cdot 1$ & $r$ & $17 / 9 V$ & تأثير مؤلفه در كروهها \\
\hline $.1 .+1$ & r & TA/TH & مؤلفهاى \\
\hline
\end{tabular}

جدول و. نتايح تحليل واريانس بين آزمودنىها و درون آزمودئىها با اندازهيرىهاى مكرر با آزمون كرين هاس كيشر

\begin{tabular}{|c|c|c|c|c|}
\hline سطح معنادارى & مقدار F م م & ميانكَين مجذورات & درجه آزادى & منيع تغييرات \\
\hline$+1+\infty 1$ & $r / A 1$ & rareq/ITV & $r$ & مؤلفهها \\
\hline$+1+\infty 1$ & RTV/GYT & TQR/RHI & $1 / \Delta V$ & مؤلفه در كروهها \\
\hline
\end{tabular}

乩

معنى دارى بهبود يافت. در متغير تعداد انتخابهاى صحيح كه

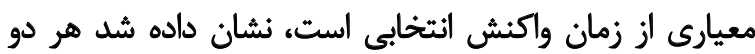

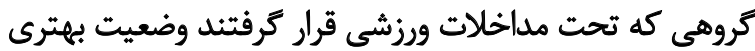

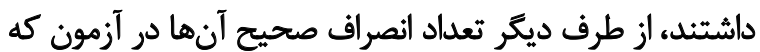

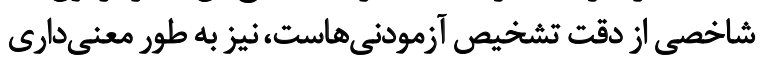

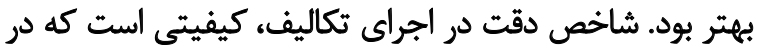

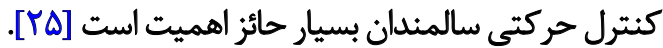
در همين راستا، نتايج اين تحقيق درباره اثربخشى تمرينات

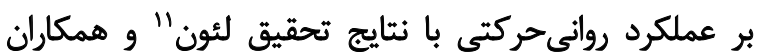
همراستاست كه نتيجه كرفتند تمرينات ادراكي حركتي موجب تئي

11. León

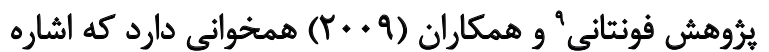

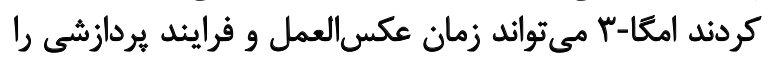

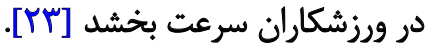
برخلاف نتايج اين تحقيق، آنتييا' و همكاران نشان دادند

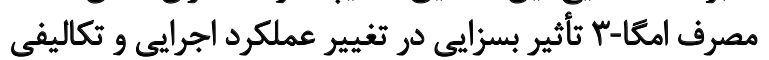

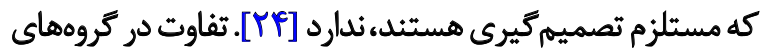

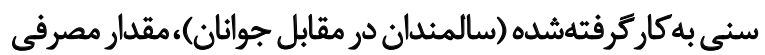
امعا-ب و طرح تحقيقى متفاوت از جمله دلايل واكرايى نتايج اين

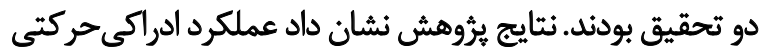

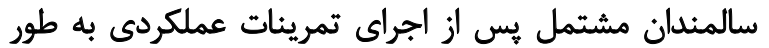

9. Fontani

10. Antypa 
مالاحظات اخلاقى

يبروى از اصول اخلاق يُوهش

طرح تحقيق به تصويب كميته اخلاق دانشكاه بينالمللى امام

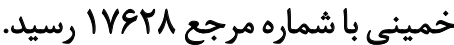

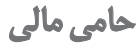

اين تحقيق از طرح يرؤشى دانشَاه بينالمللى امام خمينى به

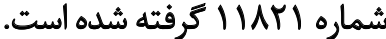

مشاركت نويسند متان

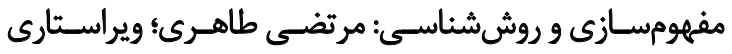

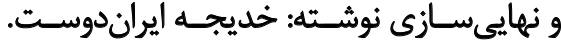

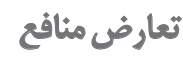

بنابر اظهار نويسندكان، اين مقاله تعارض منافع ندارد.

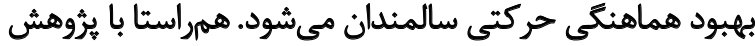

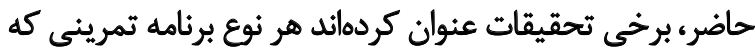

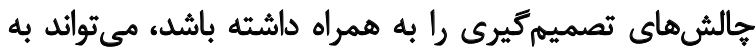

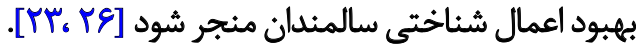

در توجيه اين نتايج مى توان به اين نكته اشاره كرد تغييرات

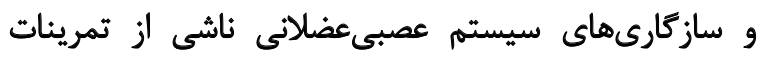

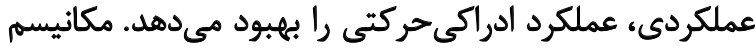

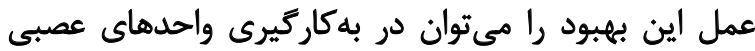

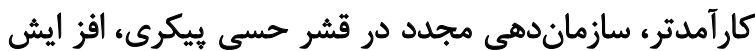

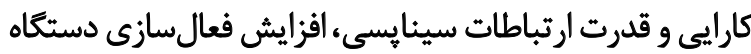

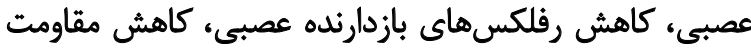

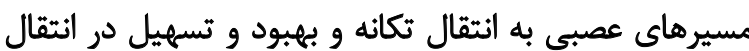

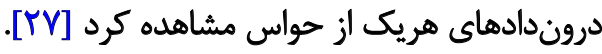

در توجيه اثربخشى امكا-r بر عمكرد روانى حركتى مي توان

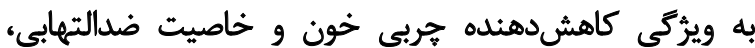

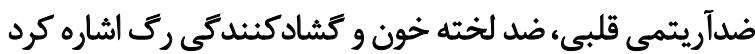

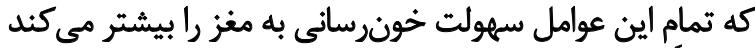

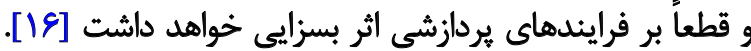

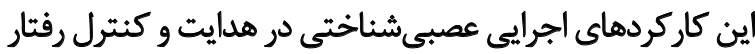

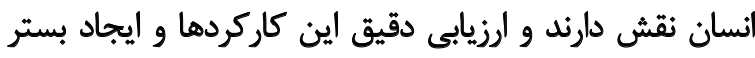

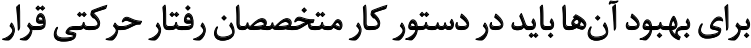

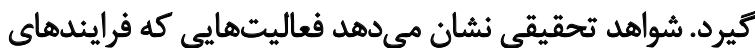

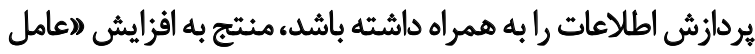

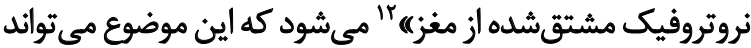

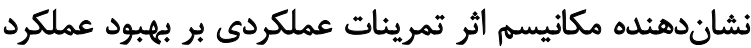
رواني حركتى باشد.

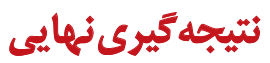

به طور كلى هركونه مداخله ورزثى كه سالمندان را از حيث

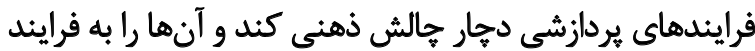

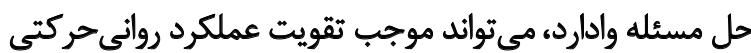

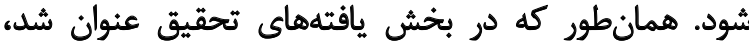

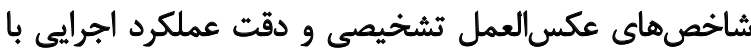

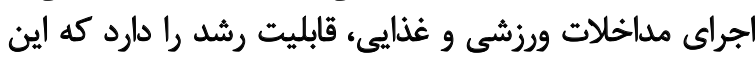

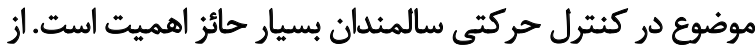

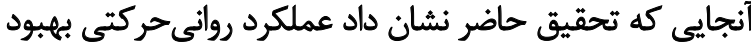

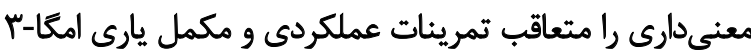

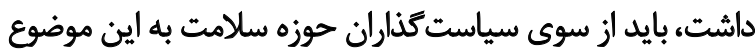




\section{References}

[1] Amini M, Mirmoezzi M, Salmanpour M, Khorshidi D. Eight weeks of aerobic exercises improves the quality of life in healthy aged sedentary men. International Journal of Sport Studies for Health. 2018; 1(1):e67514. [DOI:10.5812/ intjssh.67514]

[2] Al-Nimer M, Al-Gareeb A, Al-Kuraishy H. Omega-3 fatty acids improve psychomotor performance via mechanism not related to nitric acid production. International Journal of Green Pharmacy. 2012; 6(1):1-4. [DOI:10.4103/09738258.97102]

[3] Rodrigues-Rodrigues T, Gomes AC, Neto GR. Nutritional status and eating habits of professors of health area. International Journal of Sport Studies for Health. 2018; 1(1):e64335. [DOI:10.5812/intjssh.64335]

[4] Sharif MR, Sayyah M. Assessing physical and demographic conditions of freshman "15" male medical students. International Journal of Sport Studies for Health. 2018; 1(1):e67421. [DOI:10.5812/intjssh.67421]

[5] Monleon C, Afif AH, Mahdavi S, Rezaei M. The acute effect of low intensity aerobic exercise on psychomotor performance of athletes with nocturnal sleep deprivation. International Journal of Sport Studies for Health. 2018; 1(1):e66783. [DOI:10.5812/intjssh.66783]

[6] Jafari M, Pouryamehr E, Fathi M. The effect of eight weeks High Intensity Interval Training (HIIT) on e-selection and p-selection in young obese females. International Journal of Sport Studies for Health. 2018; 1(1):e64336. [DOI:10.5812/ intjssh.64336]

[7] Taheri M, Irandoust I. Morning exercise improves cognitive performance decrements induced by partial sleep deprivation in elite athletes. Biological Rhythm Research. 2019; 1-10. [DOI:10.1080/09291016.2019.1576279]

[8] Yates A, Norwig J, Maroon JC, Bost J, Bradley JP, Duca $\mathrm{M}$, et al. Evaluation of lipid profiles and the use of omega-3 essential fatty acid in professional football players. Sports Health. 2009; 1(1):21-30. [DOI:10.1177/1941738108326978] [PMID] [PMCID]

[9] Olusi SO, Fido AA. Serum lipid concentrations in patients with major depressive disorder. Biological Psychiatry. 1996; 40(11):1128-31. [DOI:10.1016/S0006-3223(95)00599-4] [PMID]

[10] Stunkard AJ, Faith MS, Allison KC. Depression and obesity. Biological Psychiatry. 2003; 54(3):330-7. [DOI:10.1016/ S0006-3223(03)00608-5]

[11] Morais A, Santos S, Lebre P. Psychomotor, functional, and cognitive profiles in older people with and without dementia: What connections? Dementia (London, England). 2017; 18(4):1538-53. [DOI:10.1177/1471301217719624]

[12] Faith MS, Matz PE, Jorge MA. Obesity-depression associations in the population. Journal of Psychosomatic Research. 2002; 53(4):935-42. [DOI:10.1016/S0022-3999(02)00308-2]

[13] Green KN, Martinez-Coria H, Khashwji H, Hall EB, Yurko-Mauro KA, Ellis L, et al. Dietary docosahexaenoic acid and docosapentaenoic acid ameliorate amyloid- $\beta$ and tau pathology via a mechanism involving presenilin 1 levels. Journal of Neuroscience. 2007; 27(16):4385-95. [DOI:10.1523/JNEUROSCI.0055-07.2007] [PMID]
[14] Colussi G, Catena C, Baroselli S, Nadalini E, Lapenna R, Chiuch A, et al. Omega- 3 fatty acids: From biochemistry to their clinical use in the prevention of cardiovascular disease. Recent Patents on Cardiovascular Drug Discovery. 2007; 2(1):13-21. [DOI:10.2174/157489007779606158] [PMID]

[15] Massaro M, Habib A, Lubrano L, Del Turco S, Lazzerini G, Bourcier $\mathrm{T}$, et al. The omega-3 fatty acid docosahexaenoate attenuates endothelial cyclooxygenase-2 induction through both NADP $(\mathrm{H})$ oxidase and PKC epsilon inhibition. Proceedings of the National Academy of Sciences of the United States of America. 2006; 103(41):15184-9. [DOI:10.1073/ pnas.0510086103] [PMID] [PMCID]

[16] Leslie MA, Cohen DJ, Liddle DM, Robinson LE, Ma DW. A review of the effect of omega-3 polyunsaturated fatty acids on blood triacylglycerol levels in normolipidemic and borderline hyperlipidemic individuals. Lipids in Health and Disease. 2015; 14:53. [DOI:10.1186/s12944-015-0049-7] [PMID] [PMCID]

[17] Sneddon AA, Tsofliou F, Fyfe CL, Matheson I, Jackson DM, Horgan G, et al. Effect of a conjugated linoleic acid and omega-3 fatty acid mixture on body composition and adiponectin. Obesity. 2008; 16(5):1019-24. [DOI:10.1038/ oby.2008.41] [PMID]

[18] Sato D, Seko C, Hashitomi T, Sengoku Y, Nomura T. Differential effects of water-based exercise on the cognitive function in independent elderly adults. Aging Clinical and Experimental Research. 2015; 27(2):149-59. [DOI:10.1007/ s40520-014-0252-9] [PMID]

[19] Carral JMC, Curras DM, Pérez CA, Suárez MHV. Effects of two programmes of combined Land-Based and WaterBased exercise on the cognitive function and fitness levels of healthy older adults. Motriz: Revista de Educação Física. 2017; 23(2):e101641. [DOI:10.1590/s1980-6574201700020011]

[20] Martins WR, Safons MP, Bottaro M, Blasczyk JC, Diniz LR, Fonseca RMC, et al. Effects of short term elastic resistance training on muscle mass and strength in untrained older adults: A randomized clinical trial. BMC Geriatrics. 2015; 15(1):99. [DOI:10.1186/s12877-015-0101-5] [PMID] [PMCID]

[21] Cayley P. Functional exercise for older adults. Heart, Lung \& Circulation. Heart, Lung and Circulation. 2008; 17:S70-2. [DOI:10.1016/j.hlc.2008.08.015]

[22] Dagtekin O, Gerbershagen HJ, Wagner W, Petzke F, Radbruch L, Sabatowski R. Assessing cognitive and psychomotor performance under long-term treatment with transdermal buprenorphine in chronic noncancer pain patients. Anesthesia \& Analgesia. 2007; 105(5):1442-8. [DOI:10.1213/01.ane.0000281078.65585.1e] [PMID]

[23] Fontani G, Lodi L, Migliorini S, Corradeschi F. Effect of omega- 3 and policosanol supplementation on attention and reactivity in athletes. Journal of the American College of Nutrition. 2009; 28(suppl. 4):473S-81S. [DOI:10.1080/07315 724.2009.10718114]

[24] Antypa N, Van der Does AJ, Smelt AH, Rogers RD. Omega-3 fatty acids (fish-oil) and depression-related cognition in healthy volunteers. Journal of Psychopharmacology. 2009; 23(7):831-40. [DOI:10.1177/0269881108092120] [PMID]

[25] Seghatoleslami A, Afif AH, Irandoust K, Taheri M. The impact of pilates exercises on motor control of inactive 
middle-aged women. Sleep and Hypnosis. 2018; 20(4):2626. [DOI:10.5350/Sleep.Hypn.2018.20.0160]

[26] Lehrer P, Eddie D. Dynamic processes in regulation and some implications for biofeedback and biobehavioral interventions. Applied Psychophysiology and Biofeedback. 2013; 38(2):143-55. [DOI:10.1007/s10484-013-9217-6] [PMID] [PMCID]

[27] Ahlskog JE, Geda YE, Graff-Radford NR, Petersen RC. Physical exercise as a preventive or disease-modifying treatment of dementia and brain aging. Mayo Clinic Proceedings. 2011; 86(9):876-84. [DOI:10.4016/33263.01] 
This Page Intentionally Left Blank 